\title{
ESTUDO DA PSICO-MANUTENÇÃO DA ASMA: AS COGNIÇÕES COMO PREDITORAS DE EMOÇÕES E COMPORTAMENTOS PROBLEMÁTICOS ASSOCIADOS À ASMA
}

\section{Ana Paula Soares de Matos Ana Cláudia Cardoso Machado}

\begin{abstract}
Resumo: Numa amostra de 50 doentes asmáticos hospitalizados ou em regime de consulta externa, investigou-se a associação entre as cognições disfuncionais associadas à Asma e outras variáveis psicológicas (emoções e comportamentos) tradicionalmente estudadas no âmbito dos modelos cognitivo-comportamentais, através de instrumentos de medida dessas variáveis específicos para a doença asmática. Os resultados encontrados sugerem que as cognições disfuncionais associadas à Asma são preditoras de comportamentos/emoções problemáticos que ocorrem antes, durante e depois das crises asmáticas. Sugerem-nos ainda que as cognições disfuncionais são preditoras de maiores níveis de sintomatologia "pânico/medo" e de atitudes negativas face à doença asmática e ao seu tratamento.
\end{abstract}

Palavras-chave: Cognições. Estados emocionais. Problema. Pânico. Asma.

\section{Introdução}

A doença crónica tem habitualmente uma influência muito importante no indivíduo: a) desafia a visão que este tem de si e do mundo; b) pode também activar crenças pré-existentes que induzam pensamentos negativos; $c$ ) exige uma aceitação da doença e suas consequências; e d) requer um auto-cuidado, por vezes contínuo e complexo, que permita regular o impacto e o curso da doença (Holman \& Lorig, 2000; Scandlyn, 2000). 
A Asma Brônquica é uma das doenças crónicas mais frequentes, verificando-se uma tendência para o aumento da sua incidência, apesar dos sucessivos avanços no campo da medicina e das medidas que têm vindo a ser implementadas pelos Sistemas Nacionais de Saúde (Gregerson, 2000; Comissão de Coordenação do Programa da Asma, 2000). Em Portugal, estima-se que o número de doentes ultrapasse 600.000 , sendo as taxas de prevalência mais elevadas, sobretudo, na população infantil e juvenil (Comissão de Coordenação do Programa da Asma, 2000). Além dos custos económicos, essa doença apresenta ainda custos pessoais e sociais muito elevados, pois é fonte de grande sofrimento em vários níveis, por vezes diariamente e duma forma repetida, condicionando o funcionamento quotidiano desses indivíduos.

Tradicionalmente, no estudo da doença asmática começou-se por priveligiar os seus aspectos médicos e psicossomáticos, contudo, progressivamente foi-se assistindo a um aumento do interesse na abordagem psicológica e social desses doentes.

As investigações científicas passaram a utilizar sobretudo o modelo biopsicossocial (Engel, 1977), que é por natureza um modelo sistémico, integrado, quer da saúde quer da doença, pois considera múltiplos fenómenos interactuantes (físicos, afectivos, cognitivos, comportamentais, interpessoais, socioculturais etc.) (Pereira, 2002).

Aproximando-se das novas tendências conceptuais, Kinsman, Dirks e Jones (1982) introduziram o conceito de psico-manutenção, que consideramos particularmente útil. A teoria da psico-manutenção não se refere à etiologia da doença, mas debruça-se sobre a forma como as variáveis psicológicas, sociais e comportamentais influenciam a evolução da doença, a sua gravidade e a adesão ao tratamento médico, numa perspectiva multidimensional e dinâmica.

Como profissionais de saúde, também pensamos que, no estudo da Asma Brônquica, uma abordagem interdisciplinar poderá representar a opção teórica e metodológica mais acertada se o objectivo é podermos conhecer melhor essa doença e, dessa forma, contribuir para uma efectiva melhoria da qualidade de vida dos doentes.

Entendemos que a Psicologia pode prestar um contributo importante na compreensão de algumas das variáveis do vector pessoa envolvidas nos processos de adaptação à doença. Por isso decidimos investigar algumas variáveis psicológicas relacionadas com a Asma, salientando a importância de variáveis cognitivas, emocionais e comportamentais, tradicionalmente incluídas nos modelos cognitivo-comportamentais. No presente artigo apresentamos resultados referentes à relação entre as cognições e as emoções e os comportamentos, relacionados com a Asma.

Os autores, no âmbito da Psicologia, têm amplamente referido o papel do significado na compreensão da psicopatologia (Bandura, 1986; Gui- 
dano \& Liotti, 1983, 1987; Joyce-Moniz 1997; Safran \& Segel, 1990; Safran, 1990; Young, 1990).

De acordo com o paradigma cognitivo: a) as representações que fazemos dos acontecimentos ajudam-nos a compreender as nossas respostas afectivas e comportamentais (havendo uma interacção entre pensamentos, sentimentos e comportamentos); e b) temos a potencialidade de adquirir consciência dos respectivos significados e de criar significados alternativos (Beck, 1976, 1991, 1996; Beck, Emery, \& Greenberg, 1985; Beck, Rush, Shaw, \& Emery, 1979; Beck \& Freeman, 1990; Beck \& Weishaar, 1989; Ellis, 1977; Gonçalves, 1993, 1995; Mahoney, 1991; Pinto Gouveia, 1990; Young, 1990).

Também em relação à adaptação à doença física, cada vez mais os autores pensam que a atribuição de significados é central, sendo necessário que os terapeutas conheçam as interpretações que os doentes fazem das suas experiências relacionadas com a doença e seu tratamento.

Nesse contexto, segundo White (2001), tem-se salientado a relevância da aplicação do modelo cognitivo-comportamental à doença física, particularmente à doença crónica: a) devido à centralidade que assumem nesse modelo os significados; b) uma vez que sublinha a necessidade de o doente adoptar um papel activo na relação com o terapeuta (empiricismo colaborativo), na construção dum reportório de aptidões e no auto-manejo; c) porque essas doenças habitualmente se associam com problemas psicológicos para os quais a terapia cognitivo-comportamental tem comprovada eficácia.

Sensky e Wright (1993) salientam também duas razões pelas quais a terapia cognitiva é eficaz no lidar com os distúrbios emocionais nas pessoas com doença física:a) há evidência de que as respostas individuais à doença estão relacionadas mais com factores cognitivos do que com a gravidade ou sintomas da doença; b) os problemas mais comuns são a depressão e a ansiedade - e esses são os tipos de reacções emocionais para os quais a terapia cognitiva começou por se desenvolver.

A abordagem cognitivo-comportamental tem sido aplicada com sucesso na síndrome do cólon irritável, na síndrome de fadiga crónica, na dor crónica, nas cefaleias, na doença respiratória obstrutiva crónica, no eczema atópico, na úlcera péptica, na dor pré-cordial atípica, na hipertensão, no enfarte do miocárdio, na angina de peito, no cancro (e mastectomia), na diabetes, na asma e nos doentes cirúrgicos e pode ser uma abordagem ideal para ajudar os doentes a minimizar o impacto psicológico negativo da sua doença (cf., Johnston, 1997; Keefe \& Beckham 1994; Mayou, 1997; Sensky \& Wright, 1993; Sharpe, 1997; White, 2001; para uma revisão).

Os modelos cognitivo-comportamentais realçam a importância das representações mentais e do processamento da informação (Beck, 1976, 1991, 1996; Beck, Rush, Shaw, \& Emery, 1979; J. Beck, 1995; Clark, Beck, \& Alford, 1999; Gelder, 1997; Mathews, 1997; Rachman, 1997; Teasdale, 1997). 
O modelo de Beck (Beck, 1976, 1991,1996; Beck, Rush, Shaw, \& Emery, 1979; J. Beck, 1995; Clark, Beck, \& Alford, 1999), que passamos a descrever, é uma das mais proeminentes e elaboradas teorias cognitivas que fundamenta uma abordagem psicoterapêutica amplamente aplicada, testada e reconhecida. É uma teoria esquemática, que refere a existência de vários níveis de cognição que se relacionam entre si:

a) esquemas ou crenças nucleares (são unidades estruturais da organização cognitiva, que, uma vez activadas, guiam a selecção, codificação, interpretação e avaliação dos estímulos internos e externos presentes numa situação, determinando a forma como o indivíduo a categoriza e lhe atribui significado; e que podem ser definidas como representações relativamente estáveis, globais e absolutistas que o indivíduo tem sobre si, os outros e o mundo; exemplos:"Sou um desgraçado,"'Sou vulnerável,",'Sou um fardo para toda a gente,", "Os outros têm pena de mim,",A minha doença é um drama");

b) crenças intermediárias ou suposições condicionadas, regras, atitudes (estão mais relacionadas com as situações; apresentam-se muitas vezes como regras de vida; podem ser formuladas condicionalmente ou incondicionalmente sob a forma de imperativos e proibições, podendo algumas crenças ser compensatórias de outras; exemplos: "Se não consigo controlar as crises sou um fracassado"','Se fizer o que o médico me diz deixo de ter sintomas,"'Tenho que fazer sempre o que o médico me diz","Tenho que ser sempre forte",'“Tenho que controlar tudo"');

c) distorções ou erros cognitivos (são processos cognitivos que estabelecem a ligação entre as estruturas e os produtos cognitivos e correspondem a erros ou enviesamentos no processamento da informação, quer na atenção, quer na interpretação; o indivíduo interpreta as situações distorcendo-as sistematicamente, de forma a manter os esquemas disfuncionais, mesmo perante dados contraditórios; exemplos: abstracção selectiva, pensamento dicotómico, sobregeneralização, personalização, magnificação/ minimização, inferência arbitrária, catastrofização);

d) pensamentos automáticos negativos (são produtos cognitivos; cognições com conteúdo negativo que emergem de súbito na corrente do pensamento, que podem ser interpretações de acontecimentos actuais, passados ou futuros e que se associam a emoções negativas e comportamentos; são específicos para a situação e mais fáceis de identificar do que as crenças nucleares ou intermédias; são entendidos como a porta de entrada no sistema de significações do indivíduo, pois revelam as distorções no processamento da informação e representam as crenças do indivíduo e as suas suposições; exemplos:"não consigo controlar esta crise,","agora é que vou morrer com falta de ar").

Esquemas mal-adaptativos estão na base de crenças e suposições disfuncionais. Numa situação específica essas podem ser activadas e o indivíduo faz enviesamentos no processamento da informação (distorções 
cognitivas ou erros cognitivos). Os enviesamentos na percepção e/ou interpretação da informação, após a activação de um determinado esquema disfuncional, têm a sua expressão através dos pensamentos automáticos disfuncionais ou negativos que se fazem acompanhar de emoções negativas e comportamentos e surgem sintomas de perturbações emocionais (ex.: perturbação de ansiedade).

Acontecimentos críticos (ex.: a experiência de uma doença), quando são congruentes com as crenças pré-existentes (vulnerabilidade cognitiva), podem servir de activadores, passando a informação a ser processada de uma forma particular (ex.: distorções cognitivas).

Beck, em 1996, para ultrapassar algumas insuficiências que a teoria de esquema, com o seu modelo linear (estímulo $\rightarrow$ esquema cognitivo $\rightarrow$ motivação, afecto e comportamento) apresentava na explicação dos fenómenos psicológicos e psicopatológicos, amplia-a, formulando a teoria dos modos. Os modos são conceptualizados como suborganizações da personalidade e incorporam os sistemas cognitivo, afectivo, comportamental, motivacional e, ainda, o sistema fisiológico periférico. Assim, o sistema cognitivo é composto por esquemas cognitivos, o sistema afectivo é composto por esquemas afectivos etc. A activação do modo resulta numa resposta sincronizada dos vários sistemas, para fazer face às exigências. Beck distingue ainda dois tipos importantes de modos. Os modos primitivos, que se manifestam nas reacções de sobrevivência e nos distúrbios psicopatológicos (modo de perda ou privação, modo de ameaça, modo de vítima e modo de auto-engrandecimento), e cujo processamento é rápido, automático e rígido; e modos constructivos, que estão relacionados com a capacidade para estabelecer relações íntimas, a eficácia nas relações interpessoais, esforços de realização, sentido de controlo pessoal e eficácia, criatividade, independência, e outras características que envolvem resiliência, optimismo e racionalidade (Clark, Beck, \& Alford, 1999) e cujo processamento é mais lento, consciente e estratégico.

O componente do modo que cria as condições necessárias para a sua activação chama-se esquema orientador. Os esquemas ou modos podem tornar-se hipervalentes (têm um baixo limiar de activação, são activados mais vezes, por uma vasta gama de estímulos), dominando o sistema de processamento de informação.

A formação dos esquemas depende da interacção entre padrões inatos e a experiência de vida do indivíduo (ex.: as experiências desenvolvimentais precoces com as figuras mais significativas).

Decorrente do modelo cognitivo de Beck, que descrevemos sucintamente, na Terapia Cognitiva pretende-se que os doentes adquiram formas mais eficazes de lidar com os seus pensamentos, emoções e comportamentos perturbadores. É importante que o indivíduo aprenda a modificar as suas interpretações, a relativizar as suas crenças, a desenvolver atitudes mais positivas e que tenha a oportunidade de se auto-actualizar através de 
vivências diferentes nos seus contextos socioculturais. As cognições constituem-se, assim, um ponto ideal para a intervenção (Fennell, 1989).

O modelo de Beck não é, obviamente, o único modelo cognitivo que considera a importância das representações individuais. Na tradição cognitivo-comportamental, outros modelos, como o de Lazarus (1991, 1993; Folkman \& Lazarus, 1988, 1991; Folkman et al., 1986a; Folkman, Lazarus, Gruen, \& DeLongis, 1986b; Lazarus \& Folkman, 1984) e o de Bandura (1977, 1978, 1982, 1986, 1989; Bandura, Reese, \& Adams, 1982; Bandura et al., 1985), têm servido de base a muitos estudos e intervenções em vários domínios da psicologia, particularmente na área da doença mental e física.

O modelo de auto-regulação das significações leigas da doença de Levental e colaboradores (Claudino, 2004; Leventhal, Diefenbach, \& Leventhal, 1992; Leventhal, Nerenz, \& Steele, 1984; Silva, 2003; Sousa, 2003), proeminente numa área que alguns autores têm denominado de Psicologia da Doença (ver Joyce-Moniz \& Barros, 1994, 2005, para revisão), também se tem debruçado sobre a forma como os indivíduos dão sentido e respondem à doença, salientando a importância das representações individuais e de estruturas e processos cognitivos.

Em termos de estruturas ou esquemas que são utilizados para representar as doenças, os processos sintomáticos e os processos de confronto, Leventhal e colaboradores referem a existência dos seguintes atributos representativos: identificação da doença e seus sintomas, das suas causas, da sua duração, das suas consequências e da sua controlabilidade/cura. Em termos de processos, sistematizam três regras fundamentais: simetria, tentativa de inclusão e indiferenciação.

Existe um estreito paralelismo entre o modelo de Beck e o modelo de Leventhal, como têm salientado Joyce-Moniz e Barros (1994, 2005): ambos os modelos se baseiam no paradigma do processamento da informação, utilizam o conceito de esquemas estruturais, são estratificados (com níveis mais explícitos e acessíveis e outros mais tácitos e acessíveis através da metacognição) e fazem a ligação entre as representações, a expressão emocional e os processos de confronto.

Pretendemos estudar a associação entre as cognições disfuncionais associadas à Asma e outras variáveis psicológicas específicas para a doença consideradas relevantes: comportamentos/emoções problema relacionados com a doença, sintomatologia pânico/medo, atitudes para com a doença asmática e seu tratamento.

Colocamos as seguintes hipóteses:

H1 - A variável Cognições Disfuncionais é preditora das Atitudes para com a doença asmática e seu tratamento;

H2 - A variável Cognições Disfuncionais é preditora dos Comportamentos/Emoções Problema associados à Asma;

H3 - A variável Cognições Disfuncionais é preditora da Sintomatologia Pânico/Medo. 
Efectuamos um estudo transversal, descritivo, de tipo correlacional, utilizando técnicas da estatística inferencial, nomeadamente regressão simples.

A amostra desse estudo foi constituída por 50 doentes asmáticos (40 do sexo feminino e 10 do sexo masculino), assistidos em regime de internamento e consulta externa pelos Serviços de Pneumologia e Imunoalergologia dos Hospitais da Universidade de Coimbra. Foram salvaguardadas as questões éticas da aprovação do estudo pelas entidades competentes e de consentimento informado pelos doentes que prestaram a sua colaboração duma forma anónima voluntária e anónima. Incluímos na amostra doentes em que foi diagnosticada Asma há mais de um ano e que não tinham outra patologia (psiquiátrica ou física) associada.

Em termos de gravidade da asma, 30\% dos indivíduos da amostra apresentam asma persistente ligeira, seguidos de $24 \%$ que sofrem de asma ligeira e de igual percentagem que sofrem de asma persistente moderada e de $22 \%$ que apresentam asma persistente moderada (de acordo com a classificação do G.I.N.A. - Programa Mundial para a Asma "Global Iniciative for Asthma", resultado do esforço conjunto do National Heart, Lung and Blood Institute e da Organização Mundial de Saúde).

Os doentes apresentavam idades compreendidas entre os 16 e os 73 anos. A idade média era de 40.32 anos com desvio padrão de 15.55. Para o sexo masculino a média de idade era de 37.80 anos, apresentando um desvio-padrão de 19.86, enquanto para o sexo feminino a idade média era de 41.15 anos, com um desvio-padrão de 14.47. Através da aplicação de um teste $t$ de Student verificamos que não existiam diferenças significativas entre os dois sexos, em termos de idade $(t=0.621 ; p=0.547)$.

Relativamente ao estado civil, constatou-se que a maioria dos elementos da amostra eram casados (58\%).

Nas habilitações literárias, a maior percentagem dos inquiridos, ou seja 38\%, apresentava como nível de instrução o $1^{\circ}$ Ciclo do Ensino Básico (Instrução Primária), seguidos de 20\% que possuíam o Ensino Secundário, $16 \%$ com frequência universitária e 14\% com escolaridade obrigatória.

Relativamente ao grupo socioeconómico, para a sua estratificação utilizamos a classificação de Sedas Nunes (1970), que classifica os indivíduos em 4 camadas sociais, segundo o nível de instrução e a profissão, sendo a camada I a de nível socioeconómico mais elevado e a camada IV a de nível mais baixo.

Os resultados mostraram que $22 \%$ dos nossos inquiridos estavam reformados, $18 \%$ eram estudantes, $16 \%$ pertenciam à camada social III e 16\% eram desempregados.

Os sujeitos completaram quatro questionários de auto-resposta (tipo Likert), que mediam as variáveis que pretendíamos estudar. Um deles foi construído pelas autoras de presente estudo (Inventário de Cognições Disfuncionais Associadas à Asma) e os restantes foram traduzidos e adaptados 
para a população portuguesa pelas mesmas autoras, após terem obtido autorização para o efeito junto dos autores dos respectivos questionários.

Inventário de Cognições Disfuncionais associadas à Asma, de Matos e Machado (1999). É um instrumento com 37 itens e uma única nota global, pois as análises factoriais preliminares realizadas não revelaram a existência de factores estatisticamente e teoricamente consistentes (no entanto é de salientar que o estudo dos factores continua em curso, para uma amostra maior). Obtivemos para a nota global um valor de alpha de Cronbach de .93, indicador de uma boa consistência interna. A correlação item-total corrigida revelou que a escala apresenta um bom grau de homogeneidade (a maior parte das correlações situa-se acima dos .3, sendo a mais elevada de .68). Os indivíduos da nossa amostra apresentaram uma média de 85.92 e um desvio-padrão de 23.33.

Escala de Atitudes para com a Doença e Tratamento - Respiratory IIIness Opinion Survey - RIOS, de Kinsman, Jones, Matus e Schum (1976). É uma escala de auto-avaliação constituída por 41 afirmações, contendo 7 subescalas:Optimismo - avalia em que grau cada doente realiza uma avaliação positiva da sua capacidade de fazer face à doença (os nossos doentes apresentaram ${ }^{\bar{x}}=12.68, \mathrm{DP}=2.63$ ); Visão negativa do staff clínico - Reflecte o grau de insatisfação do doente perante a hospitalização e a equipa médica habitualmente responsável pelo seu tratamento ( $\bar{x}=23.32$, DP=3.98); Especificidade da resposta inter$n a$ - trata-se de um grupo de itens não necessariamente atitudinal, mas que avalia o grau de alerta pessoal perante os sinais prodrómicos da doença ( $\bar{x}=15.08, \mathrm{DP}=3.49$ ); Controlo externo - avalia o grau em que cada doente assume a sua responsabilidade perante o tratamento ou o delega ao cuidado de outros ( ${ }^{\bar{x}}=14.42$, DP=3.98); Estigma Psicológico - avalia a tendência para ver a asma como um sinal de fraqueza psicológica ou como uma condição muito constrangedora $(\bar{x}=15, \mathrm{DP}=5.10)$; Autoritarismo face à doença e hospitalização - avalia a tendência para um estilo de funcionamento rígido e intransigente para com a doença e o tratamento $\left({ }^{\bar{x}}=19.40, \mathrm{DP}=4.68\right)$. Obtivemos valores de alpha de Cronbach entre .48 (para a Visão Negativa do Staff Clínico) e.70 (para o Estigma Psicológico). Os autores da escala obtiveram valores de alpha mais elevados, entre .68 e .84 .

Escala Revista de Comportamentos/Emoções Problema da Asma Revised Asthma Problem Behavior Checklist - RAPBC, de Creer, Wigal, Tobin, Kotses, Snyder e Winder (1989). É constituída por 76 itens que avaliam 4 dimensões: Comportamentos relacionados com a prevenção das crises ( $(\bar{x}=33.08, \mathrm{DP}=3.92)$; Comportamentos/emoções precipitan- 
tes das crises ( $\bar{x}=39.70, \mathrm{DP}=12.07)$; Comportamentos/Emoções problema vivenciados durante as crises ( $\left.{ }^{\bar{x}}=37.62, \mathrm{DP}=6.74\right)$; Comportamento/ Emoções problema consequentes das crises ( ${ }^{\bar{x}}=74.96$, DP=21.94). Encontramos valores de alpha de Cronbach entre .65 (para a dimensão comportamentos relacionados com a prevenção das crises) e .92 (para a dimensão Comportamento/Emoções problema consequentes das crises) e de .94 para a nota global. Os autores da escala obtiveram também um alpha de .94 para a nota global e um valor de teste/reteste de .80. Esses valores revelam bons índices de consistência interna e estabilidade temporal.

Lista de Sintomas da Asma (Sintomatologia Pânico-Medo) - Asthma Symptom Checklist (Panic-Fear Symptomatology) - ASCL, de Kinsman, Dahelem, Spector \& Staudenmayer (1977). Os doentes da nossa amostra apresentaram uma média de 50 e um desvio padrão de 10 e situam-se nas seguintes categorias: $42 \%$ apresentam moderada sintomatologia pânico/medo (valores das médias entre 45 e 55, de acordo com os autores da escala), 30\% apresentam sintomatologia baixa $(<45)$ e $28 \%$ apresentam sintomatologia elevada ( $>55)$. Obtivemos um alpha de Cronbach de .85 para essa dimensão. Os autores da escala encontraram um alpha de .92 .

Gostaríamos de mencionar que pensamos que os valores por nós encontrados para as características psicométricas das escalas são geralmente mais baixos do que os obtidos pelos autores das escalas, devido à nossa amostra ter um número mais reduzido de doentes, pelo que devemos entender estes dados como preliminares, pois continua em curso o estudo psicométrico para uma amostra mais alargada de doentes.

\section{Resultados}

\section{Estudo de Regressão entre as Cognições Disfuncionais e as} Atitudes para com a Doença e o Tratamento.

Do estudo da capacidade preditiva da variável Cognições Disfuncionais sobre as Atitudes para com a Doença e Tratamento, podemos constatar que aquela variável prediz significativamente as dimensões Visão Negativa do Staff Clínico e Estigma Psicológico. O Inventário de Cognições Disfuncionais explica $24.4 \%$ da variância da componente Visão Negativa do staff clínico da Escala de Atitudes e 15.3\% da variância da componente Estigma Psicológico.

Atendendo aos resultados obtidos para o coeficiente de regressão padronizado, podemos concluir que os doentes que apresentam um índi- 
ce de disfunção cognitiva mais elevado tendem a apresentar valores mais altos na Visão Negativa do Staff Clínico ( $\Omega=0.494, \mathrm{~F}=15.561, \mathrm{p}=0.000$ ) e no Estigma Psicológico $(\Omega=0.391, \mathrm{~F}=8.668, \mathrm{p}=0.005)$.

\section{Estudo da Regressão entre as Cognições Disfuncionais e os} Comportamentos-Emoções problema da Asma

Os resultados encontrados permitem-nos constatar que a variável cognições disfuncionais é preditora dos comportamentos-emoções problema da Asma, em termos globais e nos factores 2,3 e 4. A variável Cognições Disfuncionais explica $46.9 \%$ da variância do factor 4 (comportamentos) emoções problema resultantes das crises), $46.6 \%$ da nota global, $31.10 \%$ da variância do factor 2 (comportamentos/emoções problema precipitantes das crises) e $14.1 \%$ da variância do factor 3 (comportamentos/emoções problema que ocorrem nas crises). A equação de regressão tem um valor preditivo significativo: de .01 no factor 3 e de .001 nos factores 2, 4 e na nota global.

Tendo por base a interpretação dos coeficientes de regressão para os factores $2(\beta=0.570, F=23.081, p=0.000), 3(\beta=0.398 ; F=9.029, p=0.004), 4$ $(\beta=0.685 ; F=42.424 p=0.000)$ e nota global $(\beta=0.683 ; F=41.872 p=0.000)$, concluímos que os doentes que apresentam um índice de Cognições Disfuncionais mais elevado tendem a apresentar maior número de Comportamentos/Emoções Problemáticos relacionados com a doença, quer como antecedentes e consequentes das crises, quer durante as crises de Asma.

\section{Estudo de Regressão entre as Cognições Disfuncionais e a sintomatologia Pânico-Medo}

Também verificamos que o Inventário de Cognições Disfuncionais é preditor da sintomatologia pânico-medo. A análise efectuada demonstra que o Inventário de Cognições Disfuncionais explica $61.0 \%$ da variância da Sintomatologia Pânico/Medo, o que é um valor muito significativo. Com base na leitura do coeficiente de regressão padronizado $(\beta=0.781$; $F$ $=75.044 \mathrm{p}=0.000$ ), e sendo esse positivo, podemos dizer que as variáveis variam na razão directa, ou seja, os indivíduos que apresentam resultados mais elevados no Inventário de Cognições Disfuncionais apresentam também maiores resultados na Lista de Sintomas da Asma (sintomatologia pânico/medo). 


\section{Estudo das correlações entre as Cognições Disfuncionais e}

as restantes variáveis Psicológicas

Através do cálculo do coeficiente de Pearson e da aplicação do respectivo teste de significância, podemos re-avaliar a correlação existente entre as variáveis psicológicas incluídas nesse estudo e a variável cognições disfuncionais. Essa análise terá como finalidade complementar e sintetizar os resultados obtidos relativamente ao último estudo efectuado com as análises de regressão.

O Inventário de Disfunção Cognitiva correlaciona-se positivamente com as componentes Visão Negativa do Staff Clínico ( $r=0.49, \mathrm{p}<0.001)$ e Estigma Psicológico $(0.391, \mathrm{p}<0.01)$ da Escala de Atitudes para com a Doença e Tratamento, com a Escala de Comportamentos/Emoções Problema Relacionadas da Asma, na nota global $(\mathrm{r}=0.68, \mathrm{p}<0.001)$, e nos factores comportamentos e emoções problemáticas que precipitam as crises $(r=0.57, p<0.001)$, que acontecem durante as crises $(r=0.40, p<0.01)$ e que são consequentes das mesmas $(r=0.68, p<0.001)$.

\section{Discussão e Conclusões}

O complexo mecanismo de interacção entre actividade cognitiva, emoções e comportamentos é uma das características centrais da vivência humana e, por essa razão, encontra-se igualmente indissociável das experiências de saúde e de doença, influenciando, portanto, todo o processo de adaptação individual à própria doença e a sua morbilidade.

Os dados obtidos na presente investigação, na medida em que revelam uma associação positiva entre cognições disfuncionais, atitudes disfuncionais e comportamentos e emoções problemáticos relacionados com a doença, estão de acordo com os pressupostos do modelo cognitivo comportamental.

Como tivemos oportunidade de referir, esse modelo salienta que: a) a capacidade de processar informação, construir significados e elaborar representações cognitivas é fundamental para a adaptação humana; b) uma função básica do processamento de informação é a construção pessoal da realidade; c) todos os seres humanos respondem primariamente à representação cognitiva dos acontecimentos e não directamente aos acontecimentos em si; d) o processamento de informação influencia os componentes emocional, comportamental e fisiológico da experiência humana; e) os pensamentos, sentimentos e cognições são vistos como reciprocamente interactivos; f) a actividade cognitiva pode ser monitorizada; g) o disfuncionamento psicológico deve-se fundamentalmente à operação de cognições desadaptadas (Alford \& Beck, 1997; Beck, 1976; Beck, Rush, Shaw, \& Emery, 1979; Clark, Beck, \& Alford, 1999). 
Resumindo, os resultados obtidos sugerem que a variável cognições disfuncionais está associada com as restantes variáveis incluídas no nosso estudo, tendo-se observado a existência de relações positivas significativas das cognições disfuncionais com: a) as dimensões Visão Negativa do Staff Clínico e Estigma Psicológico, da Escala de Atitudes face à Doença e Tratamento; b) a Escala de Comportamentos/Emoções Problema da Asma, na nota global, e ao nível dos comportamentos-emoções problema susceptíveis de desencadear, manter e serem consequentes das próprias crises asmáticas; c) a sintomatologia pânico/medo, nos períodos de crise.

No entanto, com o presente estudo não podemos concluir que os processos cognitivos causam por si só dificuldades comportamentais e emocionais nos indivíduos asmáticos. Por um lado, existe uma ampla gama de outros factores (genéticos, biológicos, interpessoais e ambientais) que se influenciam reciprocamente e que podem aumentar a vulnerabilidade para o desenvolvimento de dificuldades comportamentais e emocionais. Por outro lado, o design transversal desta investigação reserva-nos algumas limitações no esclarecimento das relações causais que se estabelecem entre as variáveis que só poderão ser ultrapassadas por investigações longitudinais que é importante realizar em futuros estudos.

Relativamente ao cuidado metodológico que tivemos de controlar os casos que apresentassem comorbilidade com outras doenças físicas e psicológicas (enquanto critério de exclusão da amostra), os resultados de outros estudos indicam-nos que tal medida pode ter sido bastante relevante. Calam, Gregg, e Goodman (2005) encontraram que as dificuldades psicológicas encontradas nos doentes asmáticos podem resultar mais duma saúde pobre do que da Asma em si. Adams et al. (2004) obtiveram que o sofrimento/perturbações psicológicas mentais estavam associados com o estado de saúde físico nos doentes asmáticos.

Quanto à relevância das variáveis cognitivas, essa tem sido salientada por outros autores. Nos conhecidos estudos de Denver, numa amostra de cerca de 600 doentes asmáticos, aplicaram-se algumas das escalas que também utilizamos no presente estudo (RIOS, ASCL, escala Pânico/Medo) e encontrou-se que níveis moderados de ansiedade/traço e de optimismo estavam relacionados com uma resposta mais adequada à doença (em termos de utilização de recursos) (Dirks, 1978; Dirks et al., 1978; Jones, Dirks, \& Kinsman, 1980). Num estudo efectuado na Holanda por Maes e Schlosser, (1987) com 397 doentes asmáticos dos 16 aos 74 anos, que utilizou também a escala RIOS, encontraram que as cognições (estigma psicológico) e o coping dos doentes desempenham um papel determinante nas taxas de hospitalização e que o optimismo influencia positivamente o bem-estar. Carr, Leher e Hochron (1995) obtiveram .0.0 que variáveis cognitivas (cognições catastróficas acerca de sintomas corporais e cognições agorofóbicas) estavam relacionadas com pânico/medo específico para a doença (respostas de pânico/medo associadas às crises de asma, tal como nós avaliamos 
no nosso estudo). Tendo concluído que os investigadores devem explorar o uso de técnicas cognitivas como tratamento adjuvante para a asma.

Os estudos mostram igualmente a eficácia do Tratamento CognitivoComportamental para a Perturbação de Pânico combinado com a Educação para a Asma (Ross, Davis, \& MacDonald, 2005).

É importante salientar que a Perturbação de Pânico e outras perturbações psicológicas têm sido encontradas associadas à Asma, em termos de comorbilidade (Brinke et al., 2001; Cluley \& Crochame, 2001; Goodwin \& Eaton, 2005; Goodwin, Jacobi, \& Wolfgang, 2003; Goodwin \& Marusic, 2004; Katon, Richardson, \& Lozano, 2004; Stauder \& Kovacs, 2003; Vila et al., 1999).

Put et al. (2003) avaliaram um programa de mudança comportamental para a Asma (que inclui psico-educação e técnicas cognitivas e comportamentais) e obtiveram que os doentes passaram a apresentar menos sintomas, mais qualidade de vida e menos afectividade negativa.

Pensamos que os resultados por nós obtidos apontam para a importância de se desenvolverem programas de avaliação e intervenção psicológica que visem a analisar, investigar e modificar possíveis factores psicológicos que estejam a contribuir para a manutenção e/ou agravamento da doença e a dificultar todo o processo de adaptação individual à doença. Os nossos resultados sugerem-nos que, relativamente à educação e intervenção a realizar com esses doentes, se deveria dar uma ênfase particular às variáveis cognitivas, como um complemento importante às abordagens educacionais e farmacológicas tradicionais. Salienta-se por essa razão a importância da adopção de estratégias de avaliação e intervenção psicológicas, voltadas para a investigação e modificação de cognições e emoções desadaptativas e para a correcção de padrões comportamentais problemáticos, de acordo com os modelos cognitivo-comportamentais.

Terminamos salientando que consideramos que a aplicação da Terapia Cognitiva às doenças físicas requer algumas adaptações fundamentais que passamos a mencionar. a) $\mathrm{O}$ terapeuta deve ter uma relação colaborativa não só com o doente, mas também com outros profissionais envolvidos no tratamento (p.ex., médicos, enfermeiros) e a família do doente; b) deve começar por avaliar e trabalhar as cognições que o doente tem acerca do pedido de intervenção psicológica (p.ex., crenças do tipo "o meu médico desistiu de mim","Para me mandarem para o psicólogo, devem pensar que sou doido," 'O meu caso deve ser muito grave,"“Mais ninguém com asma é mandado para o psicólogo"); c) deve apresentar um modelo unificador e um racional compreensivo ao doente, que inclua aspectos psicológicos mas também biológicos inerentes à sua doença e respectivo tratamento (ou seja, que contemple as interacções recíprocas entre cognição, emoção, comportamento, processos biológicos e ambiente); d) na perspectiva psicoeducacional dos modelos cognitivos (que pode ser realizada também com a família), deve dar informação adequada que clarifique a doença, o seu tratamento e os papeis de doente e de terapeuta; deve ajudar o doente a 
perceber e a aceitar a importância dos aspectos psicológicos, nomeadamente aspectos cognitivos, na forma como lida com a sua doença, o papel de acontecimentos significativos para si (p.ex., acontecimentos estressantes) e das suas respostas cognitivas, emocionais e comportamentais; deve criar expectativas positivas em relação aos efeitos das terapias (p.ex., medicamentosa e psicológica); e) deve explorar e modificar crenças que o doente tenha sobre a sua doença, seus antecedentes, consequentes e tratamento (temas comuns são os de fraqueza pessoal, desesperança, incerteza em relação ao futuro em geral e à doença em particular, perda de controlo, diminuição da autonomia, mudanças significativas e negativas nos relacionamentos, ameaças à sobrevivência, à imagem corporal e à auto-estima.

Após a formulação do caso, elaborada conjuntamente pelo terapeuta e pelo doente, com estratégias cognitivas (de identificação e desafio de pensamentos automáticos disfuncionais, de correcção de distorções cognitivas e de modificação esquemática; através de procedimentos como auto-monitorização de pensamentos, gerar e testar hipóteses alternativas, listar de vantagens e desvantagens, diálogo socrático e descoberta guiada), combinadas com estratégias comportamentais (como realização de actividades planejadas e de tarefas graduadas, em gabinete e sobretudo em casa, para promover o ensaio comportamental), podemos conseguir que o doente melhore a sua aceitação e compreensão da doença e a sua colaboração com medidas terapêuticas necessárias à sua condição.

\title{
Study of the psycho-maintenance of Asthma: the cognitions as predictors of problematic emotions and behaviours related to Asthma
}

\begin{abstract}
In a sample of 50 asthmatic patients, either hospitalised or outpatients, we studied the relationships between dysfunctional cognitions related to Asthma and other psychological variables (emotions and behaviours) traditionally studied in cognitive-behaviour models, using specific measurement instruments of these variables for the asthmatic disease.The obtained results suggest that dysfunctional cognitions related to Asthma are predictive of problematic behaviours/emotions that occur before, during and after asthmatic crises. They suggest that dysfunctional cognitions are predictive of higher "panic/fear" symptomatology and of more negative attitudes towards asthmatic disease and treatment.
\end{abstract}

Keywords: Cognitions. Emotional states. Problematic. Panic. Asthma. 


\section{Étude du psycho-maintien de l'Asthme: les cognitions en tant que predicteurs des émotions et des comportements problématiques associés à l'Asthme}

Résumé: Dans un échantillon de 50 malades asthmatiques internés à l'hôpital ou en régime de consultation, on a étudié l'association entre les cognitions dysfonctionnelles et autres variables psychologiques (émotions, cognitions et comportements) traditionnellement étudiées chez les modèles cognitives-comportementaux, en utilisant des instruments de mesure spécifiques pour l'Asthme. Les résultats trouvés suggèrent que les cognitions dysfonctionnelles associées à l'asthme sont prédictives des comportements/émotions problématiques qui ont lieu avant, pendant et après les crises asthmatiques. Ils nous suggèrent encore que les cognitions dysfonctionnelles sont prédictives des niveaux supérieurs de symptomatologie «panique/peur » et d'attitudes négatives en ce qui concerne l'asthme et son traitement.

Mots-clés: Cognitions. États émotionnels. Problématiques. Panique. Asthme.

\section{Estudio del psico-mantenimiento de la Asma: las cogniciones comopredictoras deemocionesy deconductas problemáticas asociadas a la Asma}

Resumen: En una muestra de 50 enfermos asmáticos hospitalizados o en régimen de consulta externa, se ha investigado la asociación entre las cogniciones disfuncionales asociadas a la Asma y otras variables psicológicas (emociones y conductas) tradicionalmente estudiadas en el ámbito de los modelos cognitivo-conductuales, por medio de instrumentos de evaluación de esas variables específicos para la enfermedad asmática. Los resultados encontrados sugeren que las cogniciones disfuncionales asociadas a la Asma son predictoras de conductas y de emociones problemáticas, que ocurren antes, durante y después de las crisis asmáticas. Los datos sugeren aún que las cogniciones disfuncionales son predictoras de mayores niveles de sintomatologia "pánico/miedo" y de actitudes negativas delante de la enfermedad asmática y de su tratamiento.

Palabras-clave: Cogniciones. Estados emocionales. Problema. Pánico. Asma. 


\section{Referências}

Adams, R. J., Wilson, H. H., Taylor, A. W., Daly, A., Tursan d' Espaignet, E., Dal Grande, E., \& Ruffin, R. E. (2004). Psychological factors and asthma quality of life: A population based study. Thorax, 59, 930-935.

Alford, B. A., \& Beck, A. T. (1997). The integrative power of cognitive therapy. New York: Guilford Press.

Bandura, A. (1977). Self-efficacy: Toward a unifying theory of behaviour change. Psychological Review, 84, 191-215.

Bandura, A. (1978). The self-system in reciprocal determinism. American Psychologist, 33, 344-359.

Bandura, A. (1982). Self-efficacy mechanism in human agency. American Psychologist, $35,122-147$.

Bandura, A. (1986). Social Foundations of Thought and Action: A social cognitive approach. Englewood Cliffs NJ: Prentice-Hall.

Bandura, A. (1989). Human agency in social cognitive theory. American Psychologist, $44,1175-1184$.

Bandura, A., Reese, L. B., \& Adams, N. E. (1982). Microanalysis of action and fear arousal as a function of differential levels of perceived self-efficacy. Journal of Personality and Social Psychology, 33(1), 5-21.

Bandura, A., Taylor, C. B., Williams, S. L., Mefford, I. N., \& Barchas, J. D. (1985). Catecholamine secretion as a function of perceived self-efficacy. Journal of Consulting and Clinical Psychology, 53(3), 406-414.

Beck, A. T. (1976). Cognitive therapy and the emotional disorders. New York: International Universities Press.

Beck, A.T. (1991). Cognitive therapy: A 30-year perspective. American Psychologist (April), 368-375.

Beck, A.T. (1996). Beyond belief: A theory of modes, personality and psychopathology. In P. M. Salkovskis (Ed.), Frontiers of cognitive therapy (pp. 1-25). New York: The Guilford Press.

Beck, A.T., Emery, G., \& Greenberg, R. L. (1985). Anxiety disorders and phobias: A cognitive perspective. New York: Basic Books.

Beck, A.T., \& Freeman, A. (1990). Cognitive therapy for personality disorders. New York: Guildford.

Beck, A. T., Rush, A. J., Shaw, B. F., \& Emery, G. (1979). Cognitive therapy of depression. New York: Guilford.

Beck, A.T., \& Weishaar, M. (1989). Cognitive therapy. In A. Freeman, K. M. Simon, L. E. Beutler \& Arkowitz (Eds.), Compreensive handbook of cognitive therapy. New York: Plenum.

Beck, J. S. (1997). Cognitive therapy: Basics and beyond. New York: Guilford Press.

Brinke, A., Ouwerkerk, M. E., Zwinderman, A. H., Spinhoven, P., \& Bel, E. H. (2001). 
Psychopathology in patients with severe asthma is associated with increased health care utilization. American Journal of Respiratory and Critical Care Medicine, 163(5), 1093-1096.

Carr, R. E., Lehrer, P. M., \& Hochron, S. M. (1995). Predictors of panic-fear in Asthma. Health Psychology, 14(5), 421-426.

Calam, R., Gregg, L., \& Goodman, R. (2005). Psychological adjustment and asthma in children and adolescent: The UK Nationwide Mental Health Survey. Psychosomatic Medicine, 67(1), 105-110.

Clark, D. A., Beck, A. T., \& Alford, B. A. (1999). Scientific Foundations of Cognitive Theory and Therapy of Depression. New York: Wiley.

Claudino, A. D. (2004). O estudo das representações de doença: ajustamento emocional e adaptação funcional em pacientes com enfarte agudo do miocárdio e angina de peito. Dissertação de doutoramento apresentada à universidade de Évora.

Cluley, S., \& Crochrame, G. M. (2001). Psychological disorder in asthma is associated with poor control and poor adherence to inhaled steroids. Respiratory Medicine, 95(1), 37-39.

Comissão de Coordenação do Programa da Asma. (2000). Programa Nacional de Controlo da Asma. Direcção Geral de Saúde.

Creer, T. L., Wigal, J. K., Tobin, D. L., Kotses, H., Snyder, S. E., \& Winder, J. A. (1989). The revised asthma behavior problem checklist. Journal of Asthma, 26, 17-29.

Dirks, J.F.(1978). The psycho-maintenance of bronchial Asthma: A review and preliminary theoretical integration of panic-fear research. In Asthma, Co., Report n. 54.

Dirks, J. F., Kinsman, R. A., Horton, D. J., Fross, K. H., \& Jones, N. F. (1978). Panic Fear in Asthma. Re-hospitalization following intensive long-term treatment. Psychosomatic Medicine, 40, 5-13.

Ellis, A. (1977). The basic clinical theory of rationale-emotive therapy. In A. Ellis \& R. Grieger (Eds.), Handbook of rational-emotive therapy. New York: Springer.

Engel, G. (1977). The need for a new medical model: A challenge for biomedicine. Science, 196, 129-136.

Fenell, M. J. (1989). Depression. In K. Hawton, P. M., Salkovskis, J. Kirk \& D. M. Clark (Eds.). Cognitive behaviour therapy for psychiatric problems: A practical guide. New York: Oxford University Press.

Folkman, S., \& Lazarus, R. (1988). Coping as a mediator of emotion. Journal of Personality and Social Psychology, 54, 466-475.

Folkman, S., \& Lazarus, R. S. (1991). Coping and emotion. In A. Monat \& R. S. Lazarus (Eds.), Stress and coping, an anthology (3rd ed., pp. 207-227). New York: Columbia University Press.

Folkman, S., Lazarus, R. S., Dunkel-Schetter, C., DeLongis, A., \& Gruen, R. J. (1986a). Dynamics of a stressful encounter: Cognitive appraisal, coping and encounter outcomes. Journal of Personality and Social Psychology, 50(5), 992-1003.

Folkman, S., Lazarus, R. S., Gruen, R. J., \& DeLongis, A. (1986b). Appraisal, coping, health satus and psychological symptoms. Journal of Personality and Social 
Psychology, 50(3), 571-579.

Gelder, M. (1997). The scientific foundations of cognitive behaviour therapy. In D. M. Clark \& C. G. Fairburn (Eds.), Science and practice of cognitive behaviour Therapy (pp. 27-46). Oxford: Oxford University Press.

Gonçalves, O. F. (1993). Terapias cognitivas: teoria e prática. Porto: Afrontamento.

Gonçalves, O. F. (1995). Hermeneutics, constructivism and cognitive-behavioral therapies: From the object to the Project. In R. Neimeyer \& M. Mahoney (Eds.), Constructivism in social psychotherapy. Washington, DC.: American Psychological Association.

Goodwin, R. D., \& Eaton, W. D. (2005). Asthma, suicidal ideation, and suicide attempts: Findings from the Baltimore Epidemiologic Catchment Area Follow-up. American Journal of Public Health, 95(4), 717-722.

Goodwin, R., Jacobi, F., \& Wolfgang, T. (2003). Mental disorders and asthma in the community. Archives of General Psychiatry, 60(11), 1125-1130.

Goodwin, R. D., \& Marusic, A. (2004). Asthma and suicidal ideation among youth in the community. Crises, Journal of Crisis Intervention \& Suicide, 25(3), 99-102.

Gregerson, B. (2000). The curious 2000-Year case of asthma. Psychosomatic Medicine, 62(6), 816-827.

Guidano, V. F., \& Liotti, G. (1983). Cognitive processes and emotional disorders: A structured approach to psychotherapy. New York: Guilford.

Holman, H., \& Lorig, K. (2000). Patients as partners in managing chronic disease. British Medical Journal, 320, 526-527.

Johnston, D. W. (1997). Cardiovascular disease. In D. M. Clark \& C. G. Fairburn (Eds.), Science and practice of cognitive behaviour therapy (pp. 341-358). Oxford: Oxford University Press.

Jones, N. F., Dirks, J. F., \& Kinsman, R. A. (1980). Assessment in the psychomaintenance of chronic physical illness. Journal of Psychiatric Treatment and Evaluation, 2, 303312.

Joyce-Moniz, L. (1997). Psicopatologia do desenvolvimento do adolescente e do adulto. Lisboa: Mcgraw-Hill.

Joyce-Moniz, L., \& Barros, L. (1994). Psicologia da doença. Análise Psicológica, 2-3(12), 233.251.

Joyce-Monyz, L., \& Barros, L. (2005). Psicologia da doença para cuidados de saúde. Porto: Edições Asa.

Katon, W. J., Richardson, L., Lozano, P., \& McCauley, E. (2004). The relationship of asthma and anxiety disorders. Psychosomatic Medicine, 66(3), 349-355.

Keefe, F. J., \& Beckham, J. C. (1994). Behavioral medicine. In L. W. Craighead, W. E. Craighead, A. E. Kazdin \& M. J. Mahoney (Eds.), Cognitive and behavioral interventions (pp. 197-213). Boston: Allyn And Bacon.

Kinsman, R. A., Dirks, J. F., \& Jones, N. F. (1982). Psycho-maintenance of chronic Physical Illness: Clinical Assessment of personal styles affecting medical management. In 
T. Millon, C. Green \& R. Meagher (Eds.), Handbook of clinical health psychology. New York: Plenum.

Kinsman, R. A., Jones, N. F., Matus, I., \& Schum, R. A., (1976). Patient variables supporting chronic illness: A scale measuring attitudes toward respiratory illness and hospitalization. Journal of Nervous and Mental Disease, 163, 3, 159-165.

Kinsman, R. A., Dahlem, N. W., Spector, S. L., \& Staudenmayer, H. (1977). Observations on subjective symptomatology, coping behavior, and medical decisions in asthma. Psychosomatic Medicine, 39, 102-119.

Lazarus, R. S. (1991). Progress on a cognitive-motivational-relational theory of emotion. American Psychologist, 46(8), 819-834.

Lazarus, R. S. (1993). From psychological stress to the emotions: A history of changing outlooks. Annual Review of Psychology, 44, 1-21.

Lazarus, R., \& Folkman, S. (1984). Stress, appraisal and coping. New York: Springer.

Lehrer, P. (2002). Psychological aspects of asthma. Journal of Consulting and Clinical Psychology, 70, 3, 691-711.

Leventhal, H., Diefenbach, M., \& Leventhal, E. A. (1992). Illness cognition: Using common sense to understand treatment adherence and affect in cognition interactions. Cognitive Therapy and Research, 16(2), 143-163.

Leventhal, H., Nerenz, D., \& Steele, D. (1984). Illness representations and coping with health threats. In A. Baum, S. Taylor \& J. Singer (Eds.), A handbook of psychology and health (Vol. 4, pp. 219-252). New Jersey: Erlbaum.

Maes, S., \& Schlosser, S. (1987). The role of cognition and coping in health behaviour outcomes of asthmatic patients. Current Psychological Research and Review, 6(1), 79-90.

Mahoney, M. J. (1991). Human change processes: The scientific foundations of psychotherapy. New York: Basic Books.

Mathews, A. (1997). Information-processing biases in emotional disorders. In D. M. Clark \& C. G. Fairburn (Eds), Science and practice of cognitive behaviour therapy (pp. 47-66). Oxford University Press.

Mayou, R. (1997). Atypical chest pain. In D. M. Clark \& C. G. Fairburn (Eds.), Science and practice of cognitive behaviour therapy (pp. 359-380). Oxford: Oxford University Press.

Pereira, M.G. (2002). A perspectiva biopsicossocial na avaliação em psicologia da saúde: modelo interdependente. Psicologia: Teoria, Investigação e Prática, 2, 183-191.

Pinto Gouveia, J. A. (1990). Factores cognitivos de vulnerabilidade para a depressão: Estudo da sua interrelação com os acontecimentos de vida. Dissertação de doutoramento apresentada à Faculdade de Psicologia e de Ciências da Educação da Universidade de Coimbra.

Put, C., van den Bergh, O., Lemaigre, V., Demedts, M., \& Verleden, G. (2003). Evaluation of an individualised asthma programme directed at behavioural change. European Respiratory Journal, 21, 109-115.

Rachman, S. (1997). The evolution of cognitive behaviour therapy. In D. M. Clark \& 
C. G. Fairburn (Eds), Science and practice of cognitive behaviour therapy (pp. 1-26). Oxford University Press.

Ross, C. J., Davis, T. M., \& MacDonald, G. F. (2005). Cognitive-behavioral treatment combined with asthma education for adults with asthma and coexisting panic disorder. Clinical Nursing Research, 14(2), 131-157.

Safran, J. D. (1990). Towards a refinement of cognitive therapy in light of interpersonal theory: I. theory. Clinical Psychology Review, 10, 87-105.

Safran, J. D., \& Segal, Z. V. (1990). Interpersonal process in cognitive therapy. New York: Basic Books.

Scandlyn, J. (2000). When AIDS became a chronic disease. Western Journal of Medicine, 172, 130-133.

Sedas Nunes, A. (1970). A composição social da população portuguesa. Análise Social, 2(27-28), 331-381.

Sensky, T., \& Wright, J. H. (1993). Cognitive therapy with medical patients. In J. H. Wrigt, M. E. Tase, A. T. Beck \& J. W. Ludgate (Eds.), Cognitive therapy with inpatients: Developing a cognitive milieu (pp. 219-246). New York: Guilford.

Sharpe, M. (1997). Chronic fatigue. In D. M. Clark \& C. G. Fairburn (Eds.), Science and practice of cognitive behaviour therapy (pp. 381-414). Oxford: Oxford University Press.

Silva, I. L. (2003). Qualidade de vida e variáveis psicológicas associadas a sequelas de diabetes e sua evolução ao longo do tempo. Dissertação de doutoramento apresentada à Faculdade de Psicologia e de Ciências da Educação da Universidade do Porto.

Sousa, M. R. (2003). Estudo dos conhecimentos e representações de doença associados à adesão terapêutica nos diabéticos tipo 2. Dissertação de mestrado apresentada à Universidade de Braga.

Stauder, A., \& Kovacs, M. (2003). Anxiety symptoms in allergic patients: Identification and risk factors. Psychosomatic Medicine, 655), 816-823.

Teasdale, J. D. (1997). The relationship between cognition and emotion: the mind-inplace in mood disorders. In D. M. Clark. \& C. G. Fairburn (Eds), Science and practice of cognitive behaviour therapy (pp. 67-94). Oxford University Press.

Vila, G., Nollet-Clemençon, Vera, M., Robert, J. J., Blic, J., Jouvent, R. Mouren-Simeoni, M. C., \& Scheinmann, P. (1999). Prevalence of DSM-IV disorders in children and adolescents with asthma versus diabetes. Canadian Journal Psychiatry, 44, 562569.

White, C. A. (2001). Cognitive behaviour therapy for chronic medical problems. Chichester: Wiley.

Young, J. (1990). Cognitive therapy for personality disorders: A schema-focused approach. Sarasota, FL.: Professional Resource Exchange. 
Ana Paula Soares de Matos, Doutora em Psicologia Clínica, psicoterapeuta, Professora da Faculdade de Psicologia e de Ciências da Educação da Universidade de Coimbra. Endereço para correspondência: Rua do Colégio Novo, apartado 6153 3001-802, Coimbra, Portugal. Endereço eletrônico: apmatos@fpce.uc.pt)

Ana Cláudia Cardoso Machado, Mestre em Psicologia Clínica, membro da Associação de Desenvolvimento Integrado - Rota do Guadiana, Portugal. Endereço eletrônico: anaclaudia26@hotmail.com)

Recebido em: 1/12/2007

Aceito em: 11/08/2008 
